\title{
On Bilevel Programming and Its Impact in Branching, Cutting and Complexity
}

\author{
Andrea Lodi \\ DEIS, Università di Bologna \\ Viale Risorgimento 2, 40136 Bologna, Italy \\ andrea.lodi@unibo.it
}

\begin{abstract}
Bilevel programming is a rich paradigm to express a variety of real-world applications including game theoretic and pricing ones. However, what we are interested in this talk is to discuss the bilevel nature of two of the most crucial ingredients of enumerative methods for solving combinatorial optimization problems, namely branching and cutting.

Specifically, we discuss a new branching method for 0-1 programs called interdiction branching [3] that exploits the intrinsic bilevel nature of the problem of selecting a branching disjunction. The method is designed to overcome the difficulties encountered in solving problems for which branching on variables is inherently weak. Unlike traditional methods, selection of the disjunction in interdiction branching takes into account the best feasible solution found so far.

On the cutting plane side, we examine the nature of the so-called separation problem, which is that of generating a valid inequality violated by a given real vector, usually arising as the solution to a relaxation of the original problem. We show that the problem of generating a maximally violated valid inequality often has a natural interpretation as a bilevel program 2]. In some cases, this bilevel program can be easily reformulated as a single-level mathematical program, yielding a standard mathematical programming formulation for the separation problem. In other cases, no reformulation exists yielding surprisingly interesting examples of problems arising in the complexity hierarchies introduced by Jeroslow [1].
\end{abstract}

Keywords: Bilevel programming, branching, cutting, complexity.

\section{References}

1. Jeroslow, R.: The polynomial hierarchy and a simple model for competitive analysis. Mathematical Programming 32, 146-164 (1985)

2. Lodi, A., Ralphs, T.K., Woeginger, G.: Bilevel Programming and Maximally Violated Valid Inequalities. Technical Report OR/11/3, DEIS - Università di Bologna

3. Lodi, A., Ralphs, T.K., Rossi, F., Smriglio, S.: Interdiction Branching. Technical Report OR/09/10, DEIS - Università di Bologna 